\title{
Im Spannungsfeld unterschiedlicher Anforderungen und Interessen
}

\author{
Die Phase der Standardisierung im Nachhaltig- \\ keitsreporting hat schon vor dem CSR-Richt- \\ linie-Umsetzungsgesetz eingesetzt. Zugleich \\ stieg in den vergangenen Jahren die öffentliche \\ Aufmerksamkeit für Nachhaltigkeitsthemen. \\ Die Praxis der Berichterstattung wird schwieriger, \\ die Ergebnisse werden vielfältiger. \\ Von Sabine Braun
}

D ie Nachhaltigkeitsberichterstattung, wie wir sie lange kannten, splittet sich zunehmend in verschiedene Formate auf. Auslöser dafür sind nicht nur die unterschiedlichen Interessen der Zielgruppen von Investoren und Analysten über Kund/innen, Partner und Behörden bis hin zu Mitarbeitenden und Nachwuchs. Die Verlagerung ins Internet hat dazu ebenfalls beigetragen, ermöglicht sie doch ganz neue Formen der Ansprache und Informationsvermittlung beziehungsweise fordert diese geradezu. Nun sollte man sich angesichts der verschiedenen Formate - von PDF-Berichten über Online-Reports und Kennzahlen-Tools bis hin zu interaktiven Magazinen und Erklärvideos - aber bewusst sein, dass Nachhaltigkeitsberichterstattung schon immer wenigstens drei ganz unterschiedliche Funktionen besaß:

I Management: Die systematische Erfassung von Daten befähigt ein Unternehmen, sich einen Überblick über seine Nachhaltigkeitsleistungen zu verschaffen und Ziele für deren Verbesserung zu setzen. Regelmäßige Berichterstattung ermöglicht so strategische Steuerung.

- Transparenz: Die von den Standards der Global Reporting Initiative (GRI), dem Deutschen Nachhaltigkeitskodex (DNK) oder nun vom CSR-Richtlinie-Umsetzungsgesetz (CSR-RUG) geforderte Transparenz soll Stakeholdern mittels Managementkonzepten und Kennzahlen Einblick in das unternehmerische Handeln geben. Für nachhaltigkeitsorientierte Analysten und Investoren ist dies eine wichtige Grundlage, um Unternehmen zu bewerten, Empfehlungen zu geben und Entscheidungen zu treffen.

I Kommunikation: Damit Nachhaltigkeitsgrundsätze und -ziele die Unternehmenskultur beeinflussen und die externe Wahrnehmung des Unternehmens verändern können, müssen sie kommunikativ vermittelt werden. Eine anschauliche Darstellung dient sowohl der Sensibilisierung der Mitarbeitenden als auch der externen Reputation.
Diese Funktionen müssen nicht zwingend durch ein einziges Format abgedeckt werden. Wenn sich die Inhalte aber über zu viele Kanäle verteilen, kommt das konsistente Bild abhanden und es droht die Gefahr der Beliebigkeit. Diese Entwicklung ist aktuell zu beobachten. Treiber sind die fortschreitende Formalisierung durch CSR-RUG- und GRI-Standards und die Zunahme der öffentlichen Aufmerksamkeit für Nachhaltigkeitsthemen, die nach entsprechender Kommunikation verlangt.

\section{Standardisierung prägt die Formate}

Mit der Verpflichtung des Aufsichtsrats zur Prüfung der nichtfinanziellen Erklärung hat CSR-RUG die Aufmerksamkeit für Nachhaltigkeitsthemen in der Unternehmensleitung erhöht. Der Definition des Gesetzes nach, was wesentlich und daher zu berichten ist, sind aber nur wenige Themen relevant. Daher lässt sich das nichtfinanzielle Reporting gemäß CSRRUG meist auf wenigen Seiten und ohne Kennzahlen abhandeln. Das geschieht stark formal, an den Bestimmungen des Gesetzes orientiert. Mehr Transparenz zu gesellschaftlich relevanten Themen hat CSR-RUG damit nicht bewirkt, ja nicht einmal eine gute Berichterstattung zum Aspekt Menschenrechte, wie sie der Nationale Aktionsplan Wirtschaft und Menschenrechte (NAP) fordert.

Die meisten Unternehmen, insbesondere jene, für die nachhaltigkeitsorientierte Analysten und Investoren wichtig sind, erstellen die nichtfinanzielle Erklärung deshalb als zusätzliches Format und setzen ihre bewährte Berichterstattung gemäß GRI fort. Auch bei dieser zeigt sich eine stärkere Formalisierung, zumal wenn noch zusätzliche Frameworks wie NAP, Sustainable Development Goals (SDGs) oder die Empfehlungen der Task Force on Climate-Related Disclosures (TCFD) zu berücksichtigen sind. Nachhaltigkeitsreporting wird damit immer mehr zur Expertenberichterstattung. Die Expertenorientierung spiegelt sich oftmals in schmucklosen PDFs oder trockenen Online-Bilanzen wider. Aber auch ein Online-PDF-Bericht kann die Standardisierungserfordernisse mit einer guten Gestaltung verbinden. Dies beweist seit vielen Jahren BMW mit seinem navigierbaren und intern verlinktem PDF-Format, das seit 2018 auch den nichtfinanziellen Bericht gemäß CSRRUG umfasst.

Dass die Formate sich inzwischen oft aufteilen in Magazine mit Geschichten und schlichte GRI-Reports, liegt nicht allein an der stärkeren Zielgruppenorientierung, sondern auch an der fortwährenden Kritik der beträchtlichen Berichtsumfänge wobei: In anderen Ländern sind Nachhaltigkeitsberichte mit 
300 Seiten durchaus üblich. Natürlich lohnen sich opulent gestaltete und aufwendig getextete Berichte für nur wenige Adressaten nicht. Aber man darf schon die Frage stellen, ob sich allein mit Kennzahlen und Managementkonzepten eine glaubhafte Nachhaltigkeitspositionierung erreichen lässt. Außerdem sind auch Experten und Analysten Menschen, die anschauliche Zusammenhänge und eine gute Gestaltung schätzen, selbst wenn sie diese für ihren Zweck nicht notwendig brauchen. Erledigt haben wird sich das Thema allerdings, wenn maschinenlesbare Nachhaltigkeitsberichte Standard sind.

Wie man unterschiedliche Zielgruppenbedürfnisse parallel bedient, zeigt die REWE Group, deren Online-Nachhaltigkeitsbericht im diesjährigen Ranking der deutschen Nachhaltigkeitsberichte von IÖW und future bei den Großunternehmen auf den ersten Platz kam: Die Microsite bietet den Nutzer/innen gleich beim Einstieg die Wahl zwischen einem interaktiven Magazin und einem GRI-Bericht, der nach den Standards strukturiert ist. Magazin und Bericht sind untereinander verlinkt.

\section{Qualität braucht Zusammenhänge}

Werden Formate wie Geschichten, Kennzahlen, Rechenschaftsbericht und nichtfinanzielle Erklärung nicht nur aufgetrennt, sondern auch auf unterschiedlichen Kanälen gespielt, finden sie zwar sicher mehr Nutzer/innen, doch kann Konsistenz verloren gehen. Wenn Kennzahlen nicht mehr mit dem Kontext verlinkt sind und Geschichten nicht mehr mit Daten und Zielen korrespondieren, ist ein Gesamtzusammenhang nicht nachvollziehbar. Und gerade Sinn, Zweck und Wirkung eines Unternehmens, kommt im Auge des Betrachtenden nicht mehr zusammen.

Die Frage, wer umfassende Informationen heute überhaupt noch aufnehmen kann, ist zwar berechtigt. Dass „Schnipsel“ darauf die richtige Antwort sind, ist trotzdem zu bezweifeln. Denn wer sich wirklich interessiert, ist für Zusammenhänge dankbar. So sagte ein angesehener Nachhaltigkeitsanalyst in einem Interview zur Nachhaltigkeitsberichterstattung der Bayer AG einst, er wünsche sich grundsätzlich mehr und tiefergehende Projektbeschreibungen, um zu verstehen, wie ein Unternehmen ticke: Je mehr Informationen, desto besser. Auswählen könne man als Analyst dann selbst, das sei schließlich der Job.

Was im Kontext der Nachhaltigkeitsberichterstattung an interaktiven Formaten gestaltet und an Magazingeschichten verfasst wird, hat mit Reporting nur noch mittelbar zu tun. Es ist in bestem Sinne Kommunikation, die sich an den Bedürfnissen der Nutzer/innen und den großen Themen unserer Zeit orientiert. Klimaschutz, Menschenrechte oder Naturschutz stoßen auf große Resonanz in der Gesellschaft. Dass Unternehmen, die sich hier engagieren, davon profitieren wollen, ist legitim. Doch dafür müssen nun die Kommunikationsabteilungen und die Personalressorts gute Konzepte entwickeln, wie man Kund/innen, Mitarbeitenden und dem dringend benötigten Nachwuchs das Thema Nachhaltigkeit und das eigene Engagement nahebringt. Für die Aktivitäten beider Abteilungen ist ein umfassender Nachhaltigkeitsbericht, der Zusammenhänge beschreibt, von großem Nutzen. Denn er sichert ihren Botschaften auch bei genauerer Überprüfung Glaubwürdigkeit.

Kurzfassungen des Nachhaltigkeitsberichts oder begleitende Magazine werden heute meist auch deshalb erstellt, weil man sie bei Recruitingveranstaltungen gut auslegen kann. Den Weg, diese Informationen auch noch in einer zielgruppenadäquateren Weise darzustellen, müssen Kommunikations-, Personal- und Nachhaltigkeitsabteilungen gemeinsam gehen.

Welche Rolle die Nachhaltigkeits- oder CR-Abteilung spielt und welche Aufgaben sie hat, ist von Unternehmen zu Unternehmen - und je nach Reifegrad - verschieden. Es reicht vom verlängerten Arm der Kommunikationsabteilung über eine bei der Unternehmensstrategie oder im Umweltschutz angesie-

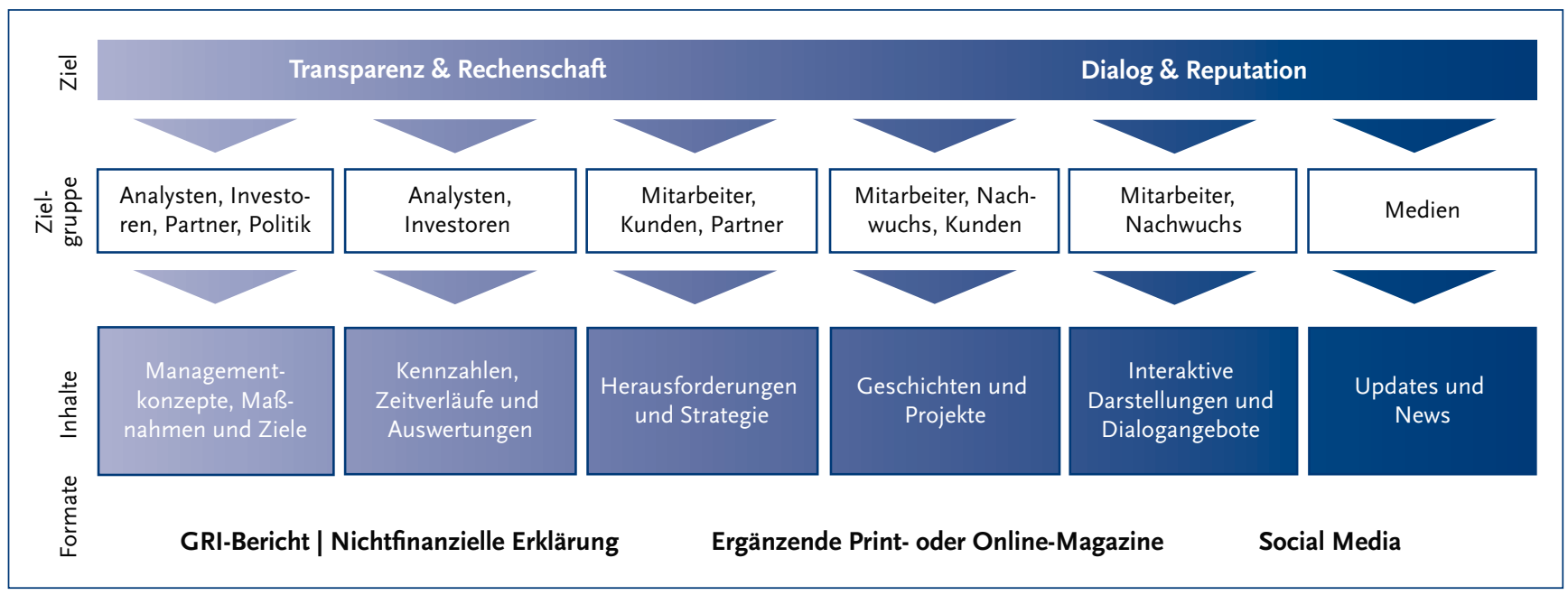

Reporting- und Kommunikationsformate 
delte Stabsstelle bis hin zur eigenen Stabsabteilung, die sich intensiv mit dem Thema Lieferkette und neuen Geschäftsmodellen auseinandersetzt. Zentrale Funktion fast aller Varianten ist aber die jährliche Erfassung der nichtfinanziellen Daten und die Erstellung des Nachhaltigkeitsberichts.

Die Umsetzung des CSR-RUG hat in fast allen Unternehmen zu Mehraufwand geführt. Diskussionen mit Fachabteilungen und vor allem mit dem Wirtschaftsprüfer kosten Zeit. Bestrebungen, das Nachhaltigkeitsreporting bei Investor Relations anzusiedeln, waren übrigens meist erfolglos. Rasch haben selbst „Newcomer“ begriffen, dass Nachhaltigkeit nicht nur eine Rechenschaftspflicht, sondern vor allem eine Managementaufgabe ist. Doch oft stehen die Nachhaltigkeitsabteilungen mit unklaren Aufgabenbeschreibungen sowie vielfältigsten und nun stetig wachsenden Anforderungen zwischen allen Fronten.

\section{Ausblick}

Was angesichts dieser Entwicklung dringend nötig wäre, ist eine Statusbestimmung und Neuausrichtung der Nachhaltigkeitsberichterstattung zwischen Regelpublizität, strategischem Controlling und Stakeholderdialog - und damit verknüpft auch eine Neudefinition der Rolle des Nachhaltigkeitsmanagements zwischen Compliance und Change. Also eine Richtungsent- scheidung darüber, ob Nachhaltigkeitsmanagement nur Risiken vermeiden, Chancen erschließen oder gar das ganze Unternehmen verändern soll.

\section{Literatur}

Deutsches Global Compact Netzwerk und econsene (Hrsg.) (2019): Neuer Impuls für die Nachhaltigkeitsberichterstattung? Studie zur Umsetzung des deutschen CSR-Richtlinie-Umsetzungsgesetzes.

\section{Briefe zur Interdisziplinarität}

\section{Geistes-, Natur-, Ingenieur- und Sozialwissenschaften, Kunst \& Handwerk im Dialog}

im oekom verlag, München. Mehr Informationen unter www.oekom.de/briefe-zur-interdisziplinaritaet
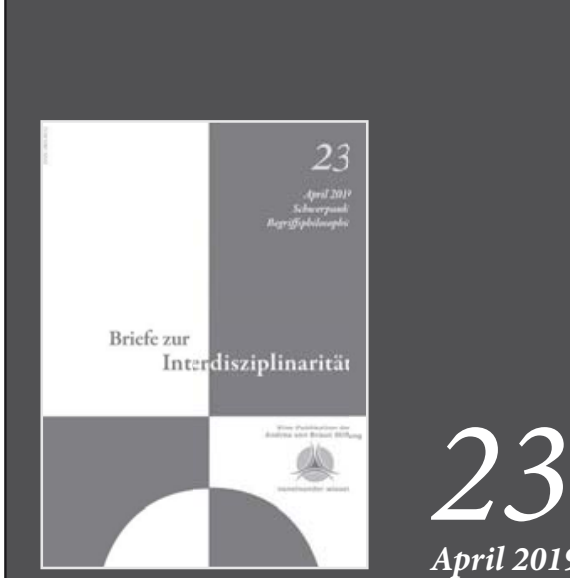

April 2019

\section{AUTORIN + KONTAKT}

Sabine Braun ist Geschäftsführerin von akzente kommunikation und beratung $\mathrm{GmbH}$. akzente kommunikation und beratung $\mathrm{GmbH}$, Corneliusstraße 10, 80469 München.

Tel.: +49 89 20205422, E-Mail: sabine.braun@akzente.de, Website: https://www.akzente.de/

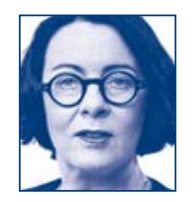

